\title{
ThD5-1
}

\section{T-ray diffraction tomography}

\author{
Bradley Ferguson ${ }^{1,2,3}$, Shaohong Wang ${ }^{1}$, Doug Gray $^{2}$, \\ Derek Abbott ${ }^{3}$ and X.-C. Zhang ${ }^{1}$ \\ ${ }^{1}$ Department of Physics, Applied Physics and Astronomy, Rensselaer Polytechnic \\ Institute, Troy, NY 12180, USA \\ ${ }^{2}$ CRC for Sensor, Signal and Information Processing, Technology Park, Mawson \\ Lakes Boulevard, Mawson Lakes, SA 5095, Australia \\ ${ }^{3}$ Centre for Biomedical Engineering and Department of Electrical \&s Electronic \\ Engineering, Adelaide University, SA 5005, Australia \\ Tel. (518) 276 3079, Fax. (518) 2766680, \\ Email.zhangxc@rpi.edu
}

T-ray computed tomography (T-ray CT) is an important new tomographic imaging modality utilizing ultrafast laser sources to probe the optical properties of 3 dimensional (3D) structures in the far-infrared [1]. It promises to have important applications in non-destructive mail and packaging inspection, semiconductor testing and manufacturing quality control. It provides sectional images of objects in an analogous manner to conventional computed tomography techniques such as X-ray CT.

An ultrafast Ti:sapphire laser is used to generate broadband pulses of THz radiation. The T-ray CT system directly measures the transmitted amplitude and phase of the $\mathrm{THz}$ field, via electro-optic sampling, at multiple projection angles and thus allows a wealth of information to be extracted from the target object including both its 3D structure and its frequency dependent far-infrared optical properties.

Using the previously described system[1] diffraction effects are neglected as the transmitted $\mathrm{THz}$ radiation is focussed and measured with a single detector. The target is scanned in $\mathrm{x}$ and $\mathrm{y}$ dimensions to determine the spatial properties of the target at each projection angle. The filtered back-projection algorithm is then used to invert the Radon transform [2] and reconstruct the 3D target.

T-ray CT works well for targets with features that are large relative to the wavelength of the $\mathrm{THz}$ radiation $(0.3 \mathrm{~mm}$ at $1 \mathrm{THz})$, however for more complex targets with fine structure the filtered back-projection algorithm is unable to accurately reconstruct the target because diffraction effects dominate the measurements. Figure 1 shows an optical photograph of a complex target. It is a piece of turkey bone which was dried in acetone for 12 hours. This sample was imaged using the T-ray CT system using 90 projection angles. The reconstructed image based on the amplitude of the measured $\mathrm{THz}$ pulses was computed using the filtered back-projection algorithm and is shown in Fig. 2. It is obvious that although the outer profile of the bone has been reconstructed with reasonable accuracy the fine internal structure is not recovered.

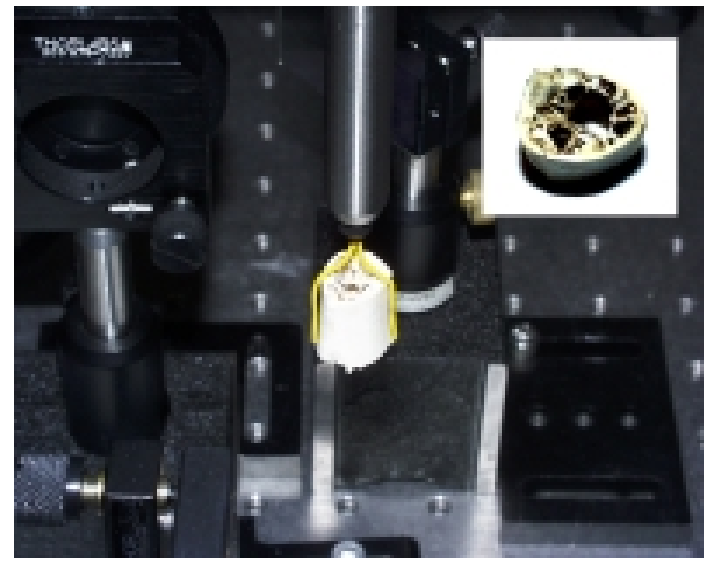

Fig. 1. A piece of turkey bone that was imaged using the scanned T-ray CT system. Inset: view of bone from above.

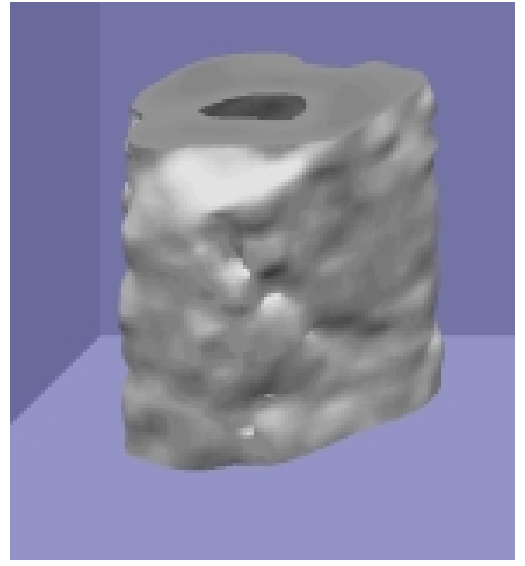

Fig. 2. Reconstructed 3D rendered image of the turkey bone. The reconstruction used the amplitude of the $\mathrm{THz}$ pulses at each pixel as the input to the filtered back-projection algorithm.

Our T-ray diffraction tomography system is similar to that described in [1]. THz pulses are generated using 


\section{ThD5-2}

a regeneratively amplified Ti:sapphire laser with an average output power of $700 \mathrm{~mW}$, a pulse duration of $130 \mathrm{fs}$ and a repetition rate of $1 \mathrm{kHz}$, incident on a biased photoconductive antenna. By using expanded $\mathrm{THz}$ and probe beams and $\mathrm{CCD}$ detection of the $2 \mathrm{D} \mathrm{THz}$ pulse [3] the need to scan the target is removed and the T-ray CT technique is accelerated by over an order of magnitude. Additionally the diffraction pattern caused by the target is observable in the measured data and can therefore aid in the object reconstruction[4]. The full data acquisition period for a projection step size of $10^{\circ}$ is approximately 8 minutes.

To test this system a simple polyethylene cylinder with a radius of $1 \mathrm{~mm}$ was imaged. Figure 3 shows the measured image for a given projection angle. The effects of diffraction are clearly visible. Reconstruction of this data using the filtered backprojection algorithm was not possible because of the diffraction effects. Instead a reconstruction based on the Born linearization of the wave equation was used [5]. This approximation is only valid for small targets but was able to reconstruct this simple target as shown in Fig. 4. For more general targets iterative methods for solving the full non-linear wave equation must be employed[6].

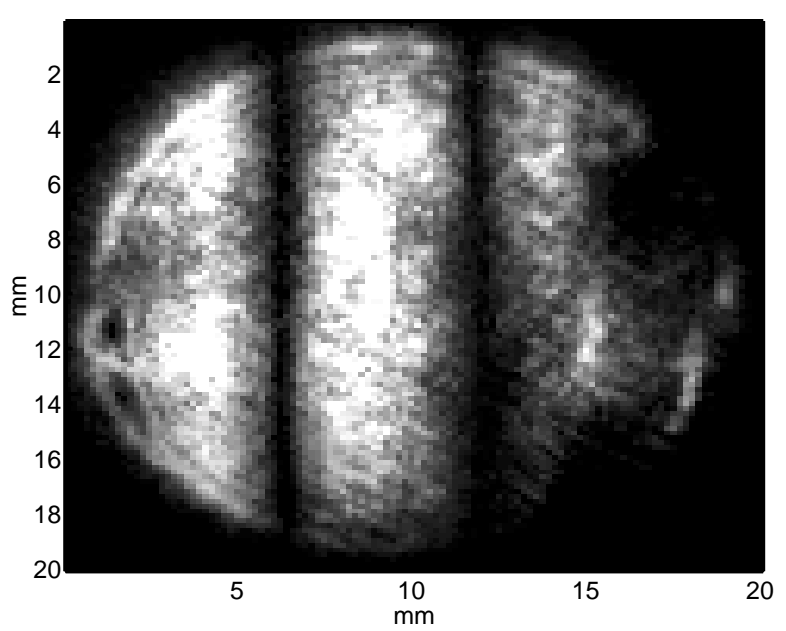

Fig. 3. THz image of a thin polyethylene cylinder for a single projection angle. The measured data was Fourier transformed and the amplitude at a frequency of $0.5 \mathrm{THz}$ is plotted. The $2 \mathrm{~cm}$ diameter circular aperture of the detector is visible as are two vertical lines resulting from diffraction of the $\mathrm{THz}$ radiation.

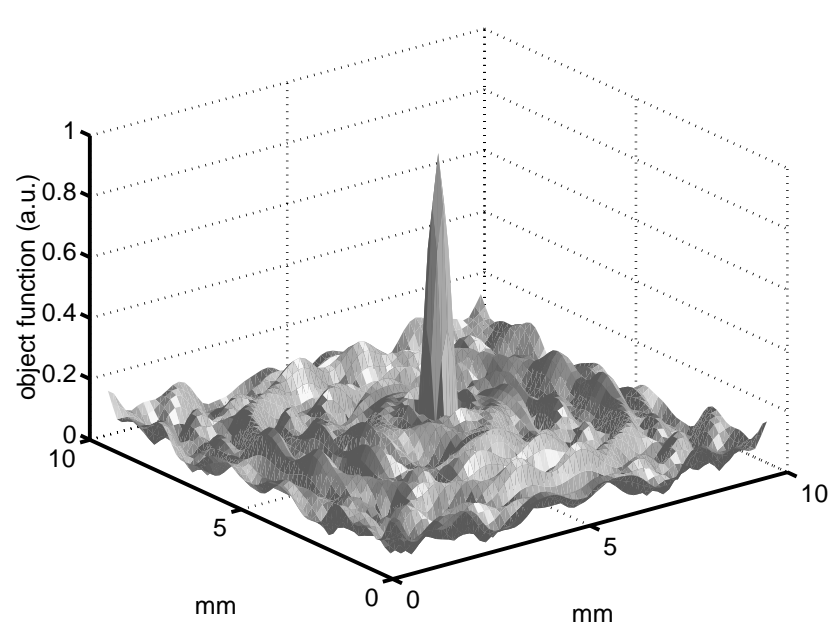

Fig. 4. Reconstructed cross-section of the polyethylene cylinder. The height shows the intensity of the reconstructed data and is related to the refractive index. The reconstruction was performed using the Born approximation and the Fourier Slice Theorem.

T-ray diffraction tomography extends the potential of terahertz time-domain spectroscopy to several new application areas. It is capable of reconstructing the 3D structure and frequency dependent far-infrared optical properties of an object, this can be performed relatively quickly by aid of $2 \mathrm{D} \mathrm{THz}$ imaging with a CCD camera. Importantly the measurement of the target's diffraction pattern may allow complex targets to be reconstructed.

\section{References}

1. B. Ferguson, S. Wang, and X. C. Zhang, "T-ray computed tomography," in 2001 IEEE/LEOS Annual Meeting Conference Proceedings, 1, pp. PD1.7-PD1.8, IEEE, (San Diego), 2001.

2. G. T. Herman, Image Reconstruction From Projections - The Fundamentals of Computerized Tomography, Academic Press, New York, 1980.

3. Q. Wu, T. D. Hewitt, and X.-C. Zhang, "Two-dimensional electro-optic imaging of terahertz beams," Applied Physics Letters 69(8), pp. 1026-1028, 1996.

4. A. B. Ruffin, J. Decker, L. Sanchez-Palencia, L. Le Hors, J. F. Whitaker, T. B. Norris, and J. V. Rudd, "Time reversal and object reconstruction with single-cycle pulses," Optics Letters 26(10), pp. 681-683, 2001.

5. A. C. Kak and M. Slaney, Principles of Computerized Tomographic Imaging, Society of Industrial and Applied Mathematics, 2001

6. F. Natterer and F. Wübbeling, Mathematical Methods in Image Reconstruction, Society of Industrial and Applied Mathematics, Philadelphia, U.S.A., 2001. 